\title{
Pathology and Therapeutics for Pharmacists: A Basis for Clinical Pharmacy Practice, $3^{\text {rd }}$ Edition Greene RJ and Harris ND (Eds).
}

Pharmaceutical Press, London (UK), 2008.

ISBN 9780853696902.

Finding just the right textbook is a challenge that many pharmacists and pharmacy students are faced with. Oftentimes we are left with little choice than to have several resources at our fingertips. Considering the volume of knowledge that is demanded of pharmacists, a resource that provides efficient access to critical information is essential. The Pathology and Therapeutics for Pharmacists: A basis for clinical pharmacy practice, is such a book.

The text is an ideal reference for pharmacists and pharmacy students. As the authors note in the preface to their first edition, "Our aim is to show the rationale and role of drug therapy in the management of some common diseases through a consideration of the mechanisms of disease processes in relation to normal function.” They note that the text is now designed to enhance patient care and management by pharmacists who now have expanded scopes of practice.

This is the third edition, and some significant updates have been made from the previous edition. The authors have included more evidence-based content and highlighted areas where guidelines should be applied. The structure has also improved, with each chapter in a consistent format, and the overall text is much easier to read, with better designed headings. There is a new chapter on haematolgoic disease and significantly expanded content in other chapters, such as a section on drugs in renal impairment found in the renal chapter.

The book is written by authors in the UK, and certainly has a focus on epidemiology, cases, and guidelines relevant to that setting. However, when international guidelines are available they are referred to in the text. There are some gaps in the text, which the authors note in the preface. For example, they highlight the most serious or most common adverse drug events, rather than providing a comprehensive list of all reported adverse effects.
Cautions and interactions are also not comprehensive. The authors emphasize the need to look up additional reference sources to confirm dosing and full product monograph information. Titles such as "Some important infections", or "Some antimuscarinics" indicate that the topics are selected, rather than comprehensive.

This text is divided into two parts. In first part, with its two chapters, the authors give a very brief outline surrounding the nature of therapeutic strategy in the first chapter while in the second chapter they discuss the principles of pathology. . The first section, titled "Basic strategy and introduction to pathology", provide a basis for the rest of the text. The first chapter includes a review of terminology, how to read/provide a case history, drug disposition, and principles of drug selection. The second chapter focuses on maladaptation as the process of disease. The authors break down pathology into four classifications, including inflammation, degeneration, neoplastic changes, and inherited disease. The remainder of the chapter then focuses on immunology (20 pages), inflammation (10 pages), and ischemia (5 pages). Although excellent reviews, the four classifications of disease are not always referred to, possibly leading to disconnect between understanding the basic disease processes and the classifications of pathological processes used by the authors.

The vast majority of the textbook is left to the second part, which contains 12 chapters. The chapter headings are arranged in the same order as the British National Formulary (BNF) (including GI and liver diseases, cardiovascular system, respiratory diseases, central nervous system, pain and its treatments, infections and antimicrobial therapy, endocrine system, neoplastic disease, haematology, rheumatology, skin disease, and the renal system). These headings will be in contrast to some textbooks, that include additional chapters, such as men's and women's health issues, 
ophthalmic conditions, etc. Each chapter starts off with a generous review of physiology that is aimed to give an awareness of how the symptoms develop. This introduction serves as a lead into the overview of the disease and its hallmarks. The authors move through etiology, pathology and pathogenesis and finally to clinical features of the disease state of interest. Now the reader is intended to be confident with tackling principles of management. These sections cover various therapeutic topics within the chapter. For example, anxiety would be a specific topic within the CNS chapter and within that are summaries such as aims and strategy, non-drug therapy, descriptions of each individual drug while finishing with recommendations on drug selection based on the clinical scenario.

In some chapters the authors present the medications from a broader perspective, versus just reviewing each drug's monograph. Each drug class is described under a general heading, such as "action", or "biopharmacy and pharmacokinetics" before moving on to the next heading, where each drug class is revisited. Such a manner of presenting information is useful for comparing medications and medication classes. This is valuable when trying to differentiate drugs from one another.

Almost every page has some sort of graphic, which complements the text and provides an easy to read reference for information. They also include tables on topics that normally would be glossed over in a sentence of two in other textbooks. For example, there is an entire table devoted to "Problems with digoxin therapy". The disease based graphics include step-wise approaches to disease. There is a number of photographs included in the skin disorders chapter.There are also "extras" that most pharmacy textbooks do not contain, such as cartoons of physical findings, and details about nonpharmacologic therapies, such as use of oxygen masks.

Readers looking for information riddled with citations and statistical graphs or tables extracted from various clinical trials will be somewhat disappointed. Indeed, the authors have acknowledged the rising dominance of evidence based medicine, nodding to major or groundbreaking studies but only when they deem it absolutely relevant. It is clear that the authors purposely chose not to break from their clear and engaging narrative style that places a tremendous emphasis on ample understanding of a disease while constantly begging the question, "Why?"

This resource provides a refreshing guide to the essentials of clinical practice. If one is looking for tables with specific half-lives, dosage ranges etc, then other resources may be more applicable. This text has the advantage of not leaving the reader overwhelmed. Rather, it is be an ideal text for students or pharmacists who have a thirst for academic support that can fill the knowledge and practice gaps.

\section{Ernest Law}

Third Year BSc Pharm Student

Faculty of Pharmacy \& Pharmaceutical Sciences University of Alberta

Edmonton, $\mathrm{AB}$

Cheryl A. Sadowski,

B.Sc.(Pharm), Pharm.D. Associate Professor Faculty of Pharmacy \& Pharmaceutical Sciences University of Alberta

Edmonton, Alberta

csadowski@pharmacy.ualberta.ca 